\title{
Extracellular Recordings of Patterned Human Pluripotent Stem Cell-Derived Cardiomyocytes on Aligned Fibers
}

\author{
Junjun Li, ${ }^{1}$ Itsunari Minami, ${ }^{1}$ Leqian Yu, ${ }^{1,2}$ Kiyotaka Tsuji, ${ }^{3}$ \\ Minako Nakajima, ${ }^{1,2}$ Jing Qiao, ${ }^{1}$ Masato Suzuki, ${ }^{3}$ Ken Shimono, ${ }^{3}$ Norio Nakatsuji, ${ }^{1}$ \\ Hitetoshi Kotera, ${ }^{2}$ Li Liu, ${ }^{1}$ and Yong Chen ${ }^{1,4}$ \\ ${ }^{1}$ Institute for Integrated Cell-Material Sciences (WPI-iCeMS), Kyoto University, Yoshida-Ushinomiya-cho, Sakyo-ku, \\ Kyoto 606-8501, Japan \\ ${ }^{2}$ Department of Micro Engineering, Kyoto University, Katsura, Nishi-ku, Kyoto 615-8540, Japan \\ ${ }^{3}$ Bio Research Department, Device Research Laboratory, Advanced Research Division, Panasonic Corporation, \\ 3-4 Hikaridai, Seika-cho, Soraku-gun, Kyoto 619-0237, Japan \\ ${ }^{4}$ Ecole Normale Supérieure, CNRS-ENS-UPMC UMR 8640, 24 Rue Lhomond, 75005 Paris, France
}

Correspondence should be addressed to Li Liu; liuli@icems.kyoto-u.ac.jp and Yong Chen; yong.chen@ens.fr

Received 10 April 2016; Accepted 29 May 2016

Academic Editor: Raymond Ching-Bong Wong

Copyright (C) 2016 Junjun Li et al. This is an open access article distributed under the Creative Commons Attribution License, which permits unrestricted use, distribution, and reproduction in any medium, provided the original work is properly cited.

\begin{abstract}
Human induced pluripotent stem cell (hiPSC) derived cardiomyocytes (CMs) hold high potential for use in drug assessment and myocardial regeneration. To create tissue-like constructs of CMs for extracellular monitoring, we placed aligned fibers (AFs) on the surface of a microelectrode array and then seeded hiPSC-CMs for subsequent monitoring for 14 days. As expected, the CMs organized into anisotropic and matured tissue and the extracellular recordings showed reduced premature beating higher signal amplitude and a higher probability of T-wave detection as compared to the culture without fibers. The CMs on the aligned fibers samples also exhibited anisotropic propagation of the field potential. These results therefore suggest that the hiPSC-CMs cultured on AFs can be used more reliably for cell based assays.
\end{abstract}

\section{Introduction}

Human cardiomyocytes (CMs) can currently be produced by differentiation of human induced pluripotent stem cells (hiPSCs) at high efficiency and high purity [1]. It is then important to form tissue-like CM constructs, rather than nonorganized cellular clusters [2-5], for more reliable drug assessment and myocardial regeneration [2-6]. In heart, cardiac tissues are formed by rod-shaped cardiomyocytes that are aligned in the form of compact and parallel myofibers with coordinated gap junctions. To mimic this organization, CMs have been cultured on patterned surfaces $[7,8]$, shrunken wrinkles [9], nanofibers [10-12], and biowires [13]. Those techniques promoted the alignment of CMs in either 2D or 3D environment, enhancing the differentiation as well as the maturation of CMs; however, the majority of these studies utilized optical calcium imaging and patch clamp techniques for functional characterization, which are invasive and inconvenient for drug assessment comparing to the extracellular recordings with microelectrode arrays.

In this study, we cultured hiPSC-CMs on aligned fibers (AFs) and followed the cardiac tissue-like construct formation. The fibers were made of polydimethylglutarimide (PMGI), which is biocompatible and can be easily electrospun [14]. Aligned PMGI fibers were produced by electrospinning and then placed by thermal transfer onto a commercial microelectrode array (MEA) to facilitate the electrophysiological monitoring of the tissue-like hiPSCCMs. As a result, compared with conventional matricescoated 2D (Flat) and random fiber- (RF-) coated substrate, we observed infiltration of CMs underneath the AF layer and the alignment of distinct sarcomeric bundles. We also observed an increased expression of cardiac maturation markers in CMs cultured on AFs comparing to the control. Accordingly, 
the extracellular recording of CMs on AFs showed reduced premature beating [15], higher signal amplitude, and higher probability of T-wave recording with respect to the control without fibers. Anisotropic propagation of the field potential was also confirmed, indicating the formation of tissue-like constructs of matured CMs and the reliability of the method for future drug screening and cardiac toxicity studies.

\section{Methods}

2.1. Fiber Fabrication and Integration. PMGI solutions with different PMGI concentrations (11\%, 13\%, 16\%, and 19\%) (MicroChem, Westborough, MA, USA) dissolved in tetrahydrofuran and cyclopentanone were prepared. For electrospinning, a direct current high-voltage generator (TechDempaz, Tsukuba, Japan) was used to provide a voltage of $8 \mathrm{kV}$. The solution was loaded into a $1 \mathrm{~mL}$ syringe, to which a needle tip of $0.6 \mathrm{~mm}$ inner diameter was attached. The positive electrode of the high-voltage power supply was connected to the needle. A grounded rotating drum was used as collector and the speed of the drum was set to $11.4 \mathrm{~m} / \mathrm{s}$ to obtain the AFs. In the case of random fibers (RFs), the drum was set to $0 \mathrm{~m} / \mathrm{s}$. The distance between the tip and the collector was maintained at $12 \mathrm{~cm}$. The humidity was measured to range from $21 \%$ to $35 \%$ while the temperature was maintained at $25^{\circ} \mathrm{C}$. Before spinning, a layer of aluminum foil was attached to the drum. The AFs were first collected by the aluminum foil, which together with the fibers was then peeled off and pressed onto a cover glass (Matsunami Glass Ind. Ltd., Kishiwada, Japan) or an MEA substrate by a Thermo Press Machine (AS ONE, Osaka, Japan). Following removal of the aluminum foil, the fibers were mostly transferred to the substrate.

2.2. Differentiation and Culture of hiPSC-CMs. hiPSCs (IMR90-1) were maintained and differentiated according to the published method [1] following the Kyoto University guidelines. After 30-50 days of differentiation, the floating colonies of CMs were collected and dissociated into single cells by stirring for $1-2 \mathrm{~h}$ in protease solution $(0.1 \%$ collagenase type I, $0.25 \%$ trypsin, $1 \mathrm{U} / \mathrm{mL}$ DNase I, $116 \mathrm{mM} \mathrm{NaCl}, 20 \mathrm{mM}$ HEPES, $12.5 \mathrm{mM} \mathrm{NaH}_{2} \mathrm{PO}_{4}, 5.6 \mathrm{mM}$ glucose, $5.4 \mathrm{mM} \mathrm{KCl}$, and $\left.0.8 \mathrm{mM} \mathrm{MgSO}_{4}[\mathrm{pH} 7.35]\right)$. After dispersion, the dissociated cells were filtered using a $40 \mu \mathrm{m}$ cell strainer (BD Falcon, Bedford, MA, USA) and resuspended in serum-supplemented cardiac differentiation medium (IMDM containing 1\% MEM nonessential amino acid solution, $1 \%$ penicillin/streptomycin, $2 \mathrm{mM} \mathrm{L-glutamine,}$ $0.5 \mathrm{mM}$ L-carnitine (Sigma-Aldrich, St. Louis, MO, USA), 0.001\% 2-mercaptoethanol, and 1-2\% BSA (Wako, Osaka, Japan), or $0.4 \%$ human serum albumin (Sigma-Aldrich)) and plated on AFs and RFs or on $0.1 \%$ gelatin-coated flat substrates (Flat) at a density of $1 \times 10^{6}$ cells $\mathrm{cm}^{-2}$. The medium was changed to serum-free medium starting from day 2 and replaced every 4 days.

Cryopreserved hiPSC-CMs (iCell) were purchased from Cellular Dynamics International (CDI) (Madison, WI, USA). The cells were thawed in prepared medium (Plating Media,
CDI) and plated on $0.1 \%$ gelatin (Sigma-Aldrich, USA) coated plates at a density of $0.5 \times 10^{6}$ cells $\mathrm{cm}^{-2}$. The medium was changed to culture medium (Maintenance Media, CDI) 2 days later. The culture medium was replaced every two days. The culture was maintained for 7 days and then replated on AF-coated or fibronectin-coated $(50 \mu \mathrm{g} / \mathrm{mL}$, Roche, Roswell,

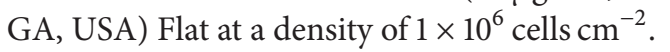

2.3. Immunostaining and Imaging. CMs derived from IMR hiPSCs were fixed with $4 \%$ paraformaldehyde and were permeabilized in PBS plus $0.5 \%$ Triton X-100 for $0.5 \mathrm{~h}$. After blocking in a 5\% normal goat serum, 5\% normal donkey serum, $3 \%$ BSA, and $0.1 \%$ Tween 20 in PBS for $16 \mathrm{~h}$ at $4^{\circ} \mathrm{C}$, the cells were incubated with an $\alpha$-actinin (mouse monoclonal IgG, 1:1000; Sigma), troponin T2 (TnT2, mouse monoclonal IgG, 1:200, Santa Cruz Biotechnology, Dallas, TX, USA), MLC2v (rabbit polyclonal IgG, 1:200; Proteintech Group, Rosemont, IL, USA), or $\beta$-MHC (mouse MYH7 monoclonal IgM, 1:100; Santa Cruz Biotechnology) antibody for $16 \mathrm{~h}$ at $4^{\circ} \mathrm{C}$. The cells were then washed and incubated with different secondary antibodies diluted in blocking buffer (1:1000): DyLight-594 anti-mouse IgG, DyLight 488 anti-mouse IgG, DyLight-594 anti-rabbit IgG, or DyLight-488 anti-mouse IgM (all from Life Technologies) at $25^{\circ} \mathrm{C}$ for $1 \mathrm{~h}$. Nuclei were visualized by DAPI (Wako).

2.4. MEA Recordings. Extracellular recordings from cultured CMs were performed by using a PC-based data acquisition system consisting of the MEA, preamplifiers, filter amplifiers (Multi Channel System, Reutlingen, Germany), data acquisition boards (ADInstruments, Dunedin, New Zealand), and software: Lab chart (ADInstruments) and MATLAB (MathWorks, Natick, MA, USA). The MEA was fabricated on a $50 \times 50 \mathrm{~mm}$ glass substrate. 60 titanium-nitride electrodes with a diameter of $30 \mu \mathrm{m}$ and distance of $200 \mu \mathrm{m}$ were organized in a $1.4 \times 1.4 \mathrm{~mm}$ matrix in the center of substrate. Cultures were stimulated using one of the electrodes. The MEA with CMs was taken from the incubator and placed on a hotplate controlled by a temperature controller maintained at $37^{\circ} \mathrm{C}$. Data were recorded at $20 \mathrm{kHz}$ with 16 -bit precision and were digitally filtered by a low pass arithmetic filter to obtain zero phase distortion with a cut-off frequency of $2 \mathrm{kHz}$. The filtered signal was differentiated digitally to determine the local activation time (LAT) at each electrode, and the activation map was constructed by interpolating the LAT values for the sites between the electrodes within the MEA matrix. The amplitude, QT interval, T-wave recording ratio, and beating rate were determined by analyzing the waveform of the CM field potential. The corrected QT interval (cQT interval) was calculated by normalization to the $\mathrm{CM}$ beating rate by using the Fridericia correction formula: $\mathrm{cQT}$ interval $=\mathrm{QT}$ interval $/ \sqrt[3]{\mathrm{RR} \text { interval. }}$.

2.5. Electron Microscopy. For scanning electron microscopy (SEM), high-resolution images were obtained using a scanning electron microscope (SEM JCM-5000; JEOL Ltd., Tokyo, Japan) operating at $10 \mathrm{kV}$. A $5 \mathrm{~nm}$ thick platinum layer was deposited on the samples by sputtering (MSP 30T; Showa 


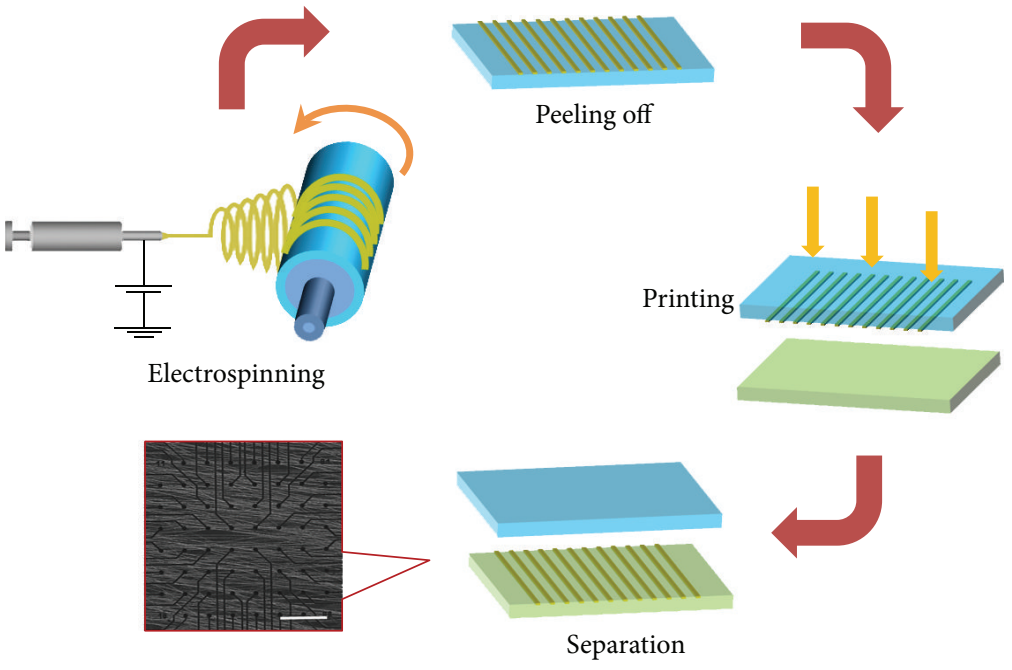

(a)

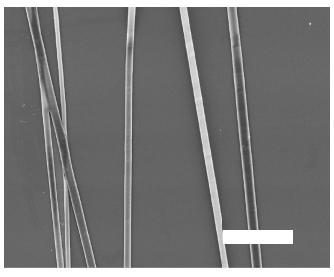

Spin time: $10 \mathrm{~s}$

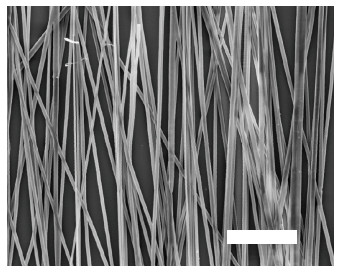

Spin time: $90 \mathrm{~s}$ (d)
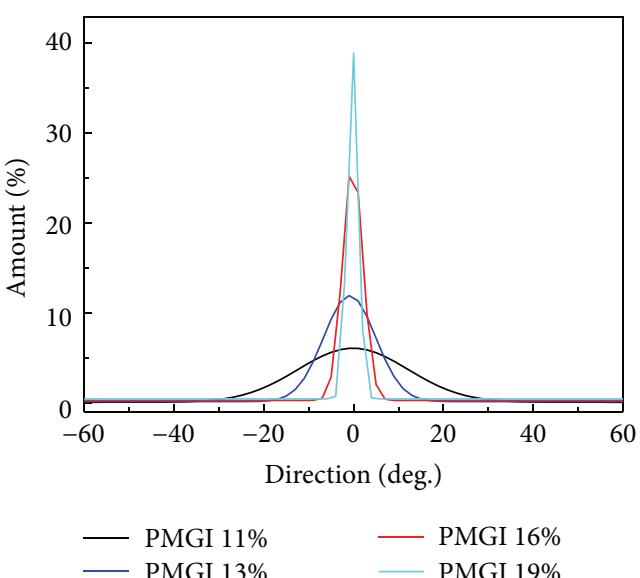

(b)

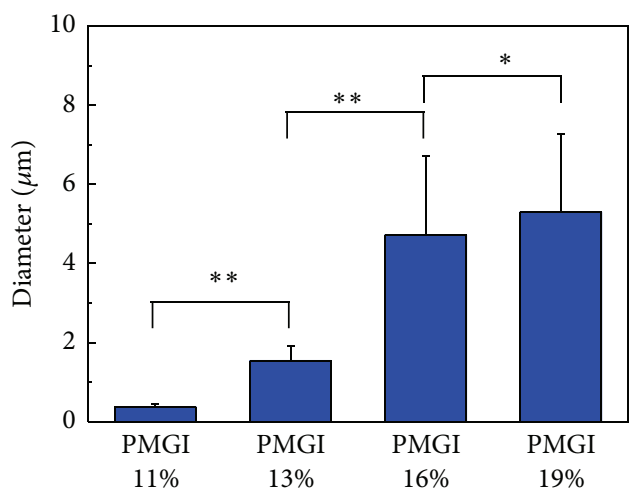

(c)

Figure 1: Preparation of aligned PMGI fibers. (a) Schematic of electrospinning and transfer of electrospun nanofibers to the surface of a microelectrode array. The scale bar $=50 \mu \mathrm{m}$. (b) Angular distributions and (c) diameter of fibers generated from PMGI of different concentrations (\%). (d) Scanning electron microscopy images of 16\% PMGI fibers electrospun for 10, 90, and $300 \mathrm{~s}$. The scale bar $=50 \mu \mathrm{m}$ (means \pm s.e., $n=3,{ }^{*} P<0.05,{ }^{* *} P<0.01$ ).

Shinku Device, Sagamihara, Japan). The angular distributions and fiber diameters were evaluated using ImageJ software (National Institutes of Health, Bethesda, MD, USA).

For transmission electron microscopy (TEM), the samples were fixed with $2 \%$ glutaraldehyde (Distilled EM Grade, Electron Microscopy Sciences, Hatfield, PA, USA) in NaHCa buffer $\left(100 \mathrm{mM} \mathrm{NaCl}, 30 \mathrm{mM}\right.$ HEPES, $2 \mathrm{mM} \mathrm{CaCl}_{2}$, adjusted to $\mathrm{pH} 7.4$ with $\mathrm{NaOH}$ ) and then postfixed with $0.25 \%$ osmium $/ 0.25 \% \mathrm{~K}_{4} \mathrm{Fe}(\mathrm{CN})_{6}$, with $1 \%$ tannic acid and finally with $50 \mathrm{mM}$ uranyl acetate. The samples were then washed, dehydrated in a series of ethanol solutions, and embedded in TABA EPON 812 resin (TAAB Laboratories Equipment Ltd., Reading, UK). After polymerization at $65^{\circ} \mathrm{C}$, ultra-thin sections $(60-100 \mathrm{~nm})$ were cut vertical to the PMGI fiber orientation using an ultramicrotome (Leica FC6, Vienna, Austria). The sections were then mounted on EM grids, stained with lead citrate, and observed by TEM (JEOL JEM1400).

2.6. Cell Attachment Assay. IMR hiPSC-CMs were seeded on fibers or other substrates at a density of $10^{6}$ cells $\mathrm{cm}^{-2}$ for $5 \mathrm{~h}$, and after the samples were rinsed twice with PBS, the adherent cells were harvested and counted. The cell attachment rate was evaluated using the following equation: cell attachment rate $(\%)=$ number of adhered cells $\times 100 /$ number of seeded cells.

2.7. Statistical Analysis. All quantitative data are presented as the means \pm standard error of the means. The difference between two groups was analyzed by Student's $t$-test and $P<$ 0.05 was considered statically significant.

\section{Results and Discussion}

3.1. Preparation of Aligned PMGI Fibers. Electrospun fibers have been often used to guide the orientation of various types of cells [16]. Here, aligned PMGI fibers were obtained by electrospinning with a rotating drum (Figure 1(a)). The alignment and diameter of fibers could be controlled by changing the concentration of PMGI (Figures 1(b) and 1(c)). The 19\% and $16 \%$ PMGI fibers demonstrated the best alignment compared to those from lower concentration PMGI. However, at the highest concentration (19\%) the PMGI solution exhibits a higher viscosity, resulting in a fiber diameter larger than $5 \mu \mathrm{m}$ that made transfer onto the MEA surface difficult. In contrast, the $16 \%$ PMGI fibers could be easily transferred to the MEA 


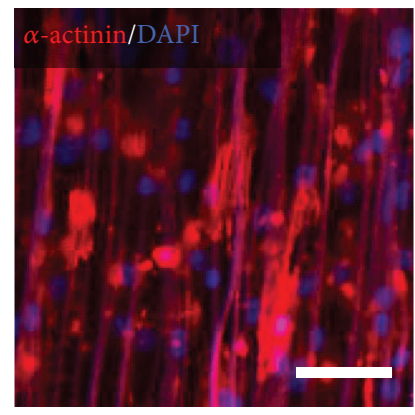

(a)

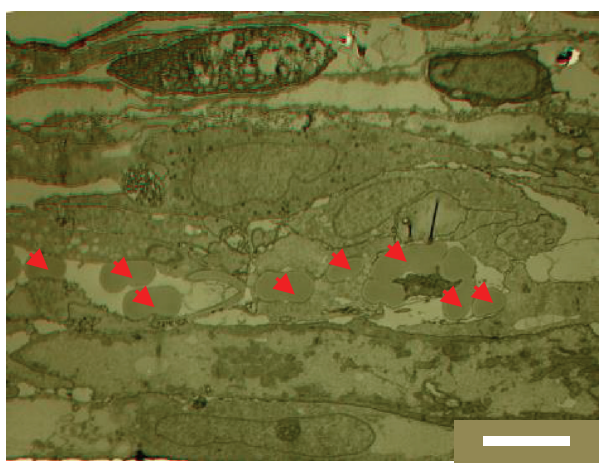

(e)

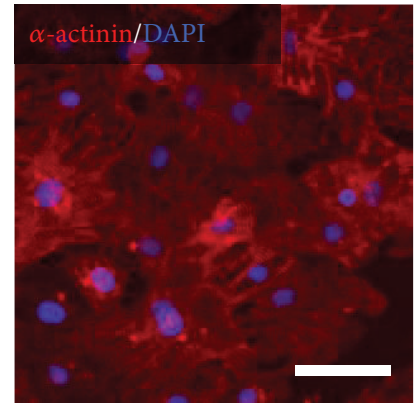

(b)

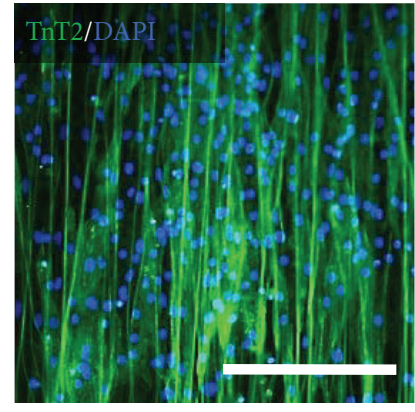

(c)

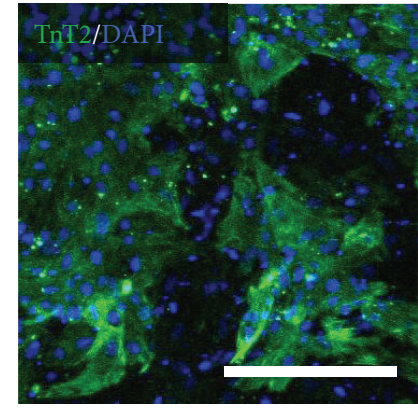

(d)

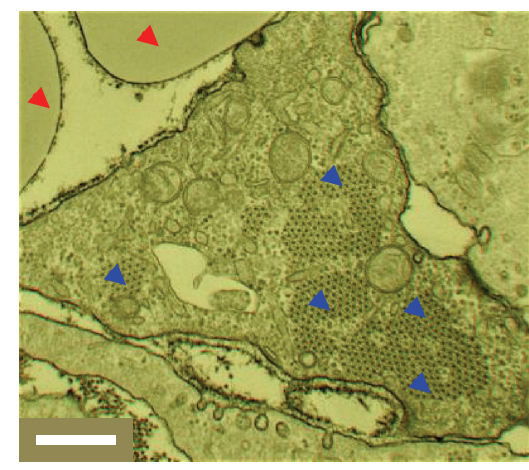

(f)

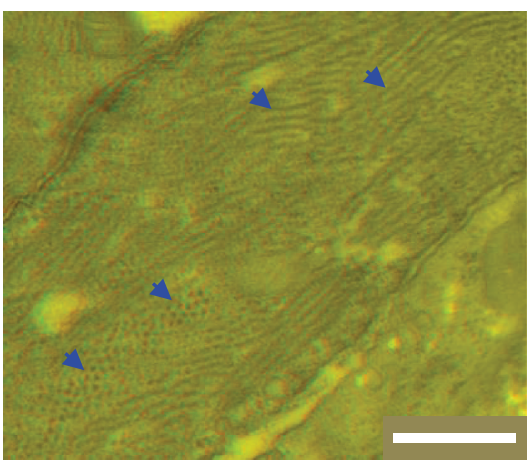

(g)

FIGURE 2: IMR hiPS-cardiomyocytes (CMs) on PMGI fibers. (a-d) Fluorescence image of CMs cultured for 14 days on aligned fibers (AFs) and gelatin-coated flat substrates (Flat). (a,b) $\alpha$-actinin is labeled in red and (c-d) TnT2 in green. The color on fiber is due to autofluorescence of PMGI. The scale bar in $(\mathrm{a}, \mathrm{b})=50 \mu \mathrm{m}$. The scale bar in $(\mathrm{c}, \mathrm{d})=200 \mu \mathrm{m}$. (e) Transmission electron microcopy images of CMs cultured for 14 days on AFs. The red arrows mark the fibers. The scale bar $=5 \mu \mathrm{m}$. (f, g) Higher magnitude TEM images of CMs cultured for 14 days on AFs (f) and Flat (g). The red and blue arrows mark the fibers and the sarcomeric bundles, respectively. The scale bar $=500 \mathrm{~nm}$.

surface owing to the relatively smaller fiber diameters. The density of the fiber sheets could be manipulated by varying the spinning time (Figure 1(d)). Typically, we obtained fibers ranging from a sparse distribution $10 \mathrm{~s}$ spinning to a thick fiber layer after $300 \mathrm{~s}$ spinning, which might prohibit the infiltration of CMs and impede the contact with the electrodes underneath the fibers. Therefore, 90 s electrospun fibers were chosen for CM culture. The as-spun PMGI fibers were then transferred to the surface of the MEA, which enabled the extracellular recording of CM activities.

\subsection{CMs Cultured on the PMGI Fibrous Substrate. The} IMR hiPSC-CMs were seeded on PMGI fibers and gelatincoated Flat for attachment tests. At $5 \mathrm{~h}$ after seeding, the samples were rinsed with PBS to remove the unattached cells. A significant difference of $\mathrm{CM}$ attachment was observed between the three types of substrates, which suggested the reliability of using PMGI fibers (Supplementary Figure 1 in Supplementary Material available online at http://dx.doi.org/10.1155/2016/2634013). Next, immunocytochemical analysis was carried out after seeding CMs on different substrates at day 14 (Figures 2(a)-2(d)). As shown in Figure 2(a), CMs on AF showed elongated shape and preferential orientation in the direction of the AFs. The alignment of the PMGI fibers clearly influenced the orientation of sarcomeric $\alpha$-actinin in the CMs. When the cells were cultured on RF and Flat, the $\alpha$-actinin filaments appeared disordered and scattered in all directions (Figure 2(b) and Supplementary Figure 2(a)). This could also be observed in another cardiomyocyte specific marker expression, TnT2 (Figures 2(c) and 2(d)).

Previously, scaffolds generated by electrospinning tended to have a pore size smaller than $10 \mu \mathrm{m}$ so that the cells could not easily infiltrate the fibers and form 3D tissue such as the extracellular matrix under in vivo conditions [17]. In comparison, in this study, after culture for 14 days, the CMs on the AFs were examined with TEM. Notably, the AFs appeared to have been embraced by the CMs, indicating that the CMs could effectively infiltrate into the area underneath the fibers and organized tissue-like structure with multilayers (Figure 2(e)). Furthermore, the oriented fibers led to the alignment of the huge sarcomeric bundles in the AF samples; these were all vertical to the observing plane (Figure 2(f)). In comparison, randomly arranged sarcomeric bundles, vertical or parallel to the observing plane, could be observed in the RF and Flat sample (Supplementary Figures 2(b) and 2(g)).

3.3. AFs Promote the Maturation of hiPSC-CMs. We next compared the effect of IMR hiPSC-CMs on MEA devices with or without AFs (Figure 3(a)). In addition, CMs were used for extracellular recording. As shown in Figure 3(a), CMs on AF-coated MEA exhibited elongated shapes and more 
AF

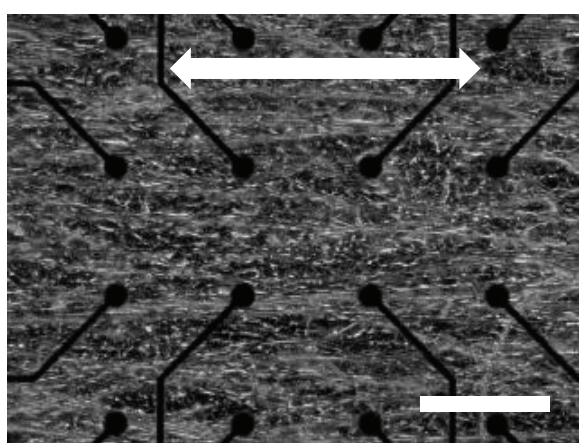

(a)

AF
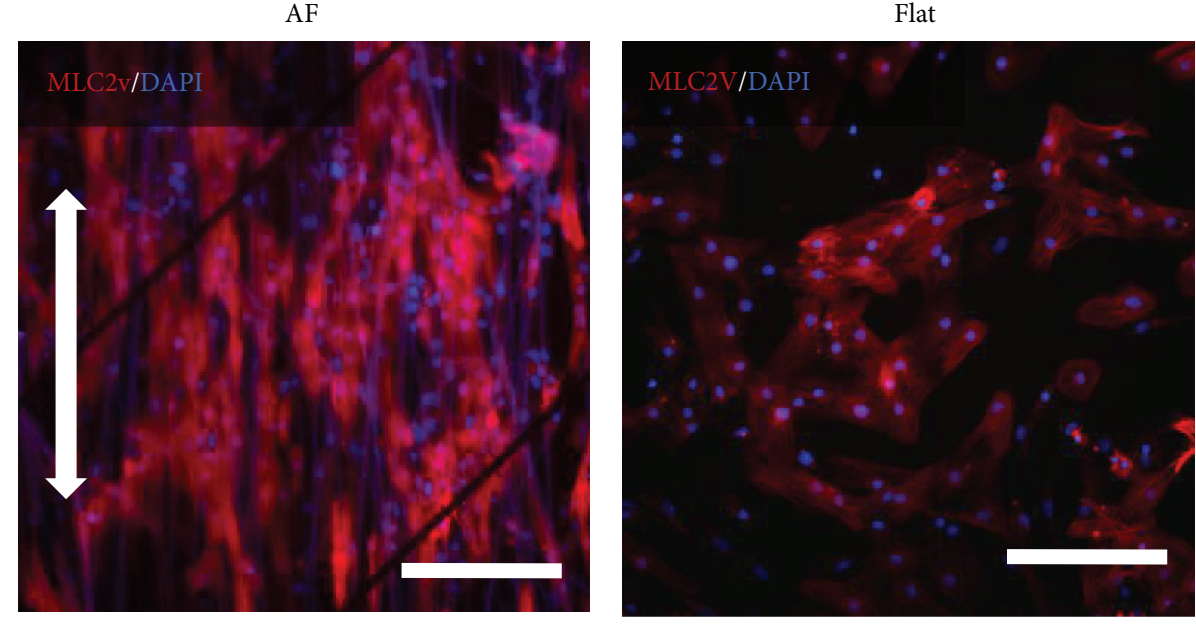

(b)

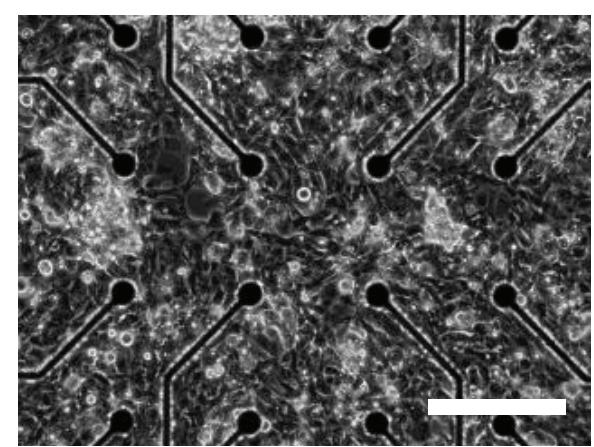

)

Flat
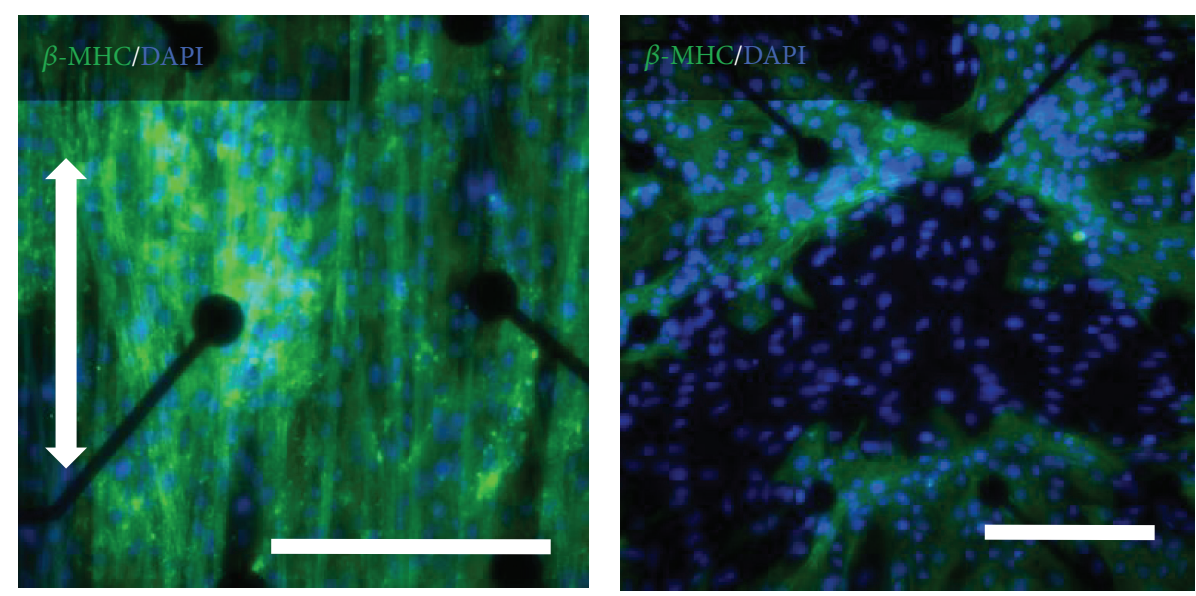

(c)

FIGURE 3: IMR hiPS-cardiomyocytes (CMs) cultured on aligned fiber (AF)-coated and gelatin-coated (Flat) microelectrode arrays (MEA). (a) Phase contrast images of CMs plated on the MEA for 2 days: the scale bar in $=200 \mu \mathrm{m}$. (b, c) Fluorescence images of CMs cultured for 14 days on MEA. (b) MLC2v is shown in red, (c) $\beta$-MHC in green, and DAPI in blue. The scale bar $=200 \mu \mathrm{m}$. The color on fiber is due to autofluorescence of PMGI. White arrow marked the fiber orientation. 
homogeneous morphology than those on the RF and Flat sample. Notably, immunostaining for cardiac tissue-specific markers indicated that significantly more cells were positive in AF samples for MLC2v (Figure 3(b) and Supplementary Figure 2(c)), a marker related to ventricular structures, and $\beta$ MHC (Figure 3(c) and Supplementary Figure 2(d)), a cardiac maturity marker correlated with contractile velocity [18]. These results indicated the efficiency of AFs in mediating CM organization and maturation than the RF and Flat control.

3.4. Electrophysiological Profile of CMs on the PMGI Fibrous Substrate. CMs are electrically active cells. Extracellular recording of spontaneous/stimulated electrical activity in contracting CMs has been shown to enable the assessment of the electrophysiological features of the CMs (Supplementary Figure 3) [19]. Right now, the researches on hiPSCCMs-based drug assessment are all utilizing the MEA with fibronectin-coated, Matrigel-coated, or gelatin-coated surface $[5,15,20,21]$, and the CMs on RF-coated substrate showed no better organization or maturation than those in Flat sample despite of their enhanced attachment on RFs; we thus chose the Flat as the control in the following electrophysiology assessment.

In addition to the observation of spontaneous beating at day 6 (Figures 4(a) and 4(b)), we recorded premature beats within some of the Flat samples (Figures 4(c) and 4(d)) indicating lower synchronization among the IMR hiPSCCMs in the Flat samples. In contrast, no premature beats were recorded with AF samples, indicating reliable intercellular connectivity and coupling of CMs in the tissue-like constructs, which can be also confirmed by the dramatically higher amplitude of recorded signal on day 6 (Figure 4(e)). The differences in connectivity and coupling between the two types of samples could be seen more clearly in the activation maps drawn based on the spontaneous contractility (Figures 5(a) and 5(b)). Here, CMs in the Flat samples showed a regional conduction delay but no such effect was observed in the AF sample. Furthermore, we observed a higher amplitude of field potential (FP) from the CMs plated on AFs compared to those on Flat during 14 days, which indicated a better cell attachment on AF-coated MEA surfaces (Figures 5(c) and $5(\mathrm{~d})$ ). The same result was confirmed by using cultured commercial CMs (iCell) on two types of substrates (Supplementary Figures 4(a) and 4(b)).

Moreover, we evaluated the percentage of the channels that had a recorded $\mathrm{T}$-wave and identified a much higher recording ratio with the AF samples compared to the Flat samples (Figure 5(e) and Supplementary Figure 4(c)). Since the T-wave recording is important for determining the QT interval variation during drug assessment, our results suggest not only a better cellular attachment but also more robust signal readout with the CMs cultured on AF-coated MEA surfaces. On the other hand, we noted higher T-wave amplitude of IMR hiPSC-CMs than of iCell (Figure 5(c) and Supplementary Figure 4(a)). The cQT interval showed no obvious difference between IMR hiPSC-CMs in AF and Flat samples (Figure 5(f)). However, the IMR hiPSC-CMs showed dramatically shorter QT intervals than the iCell (Figure 5(c)

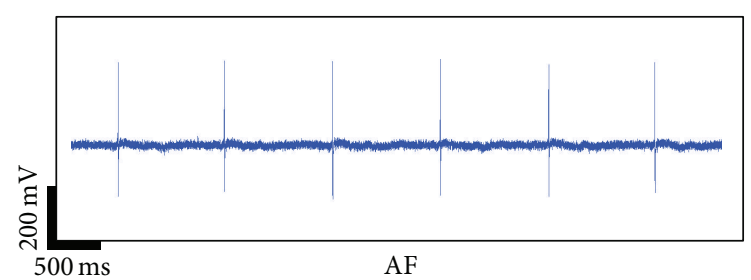

(a)

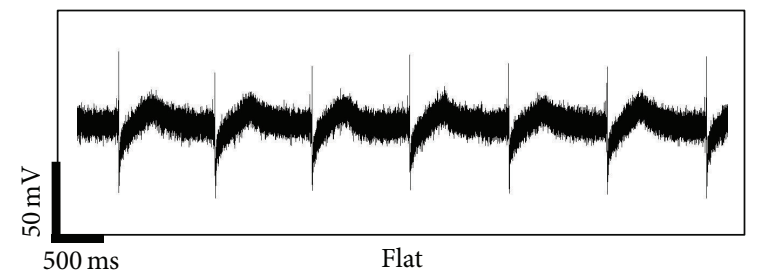

(b)

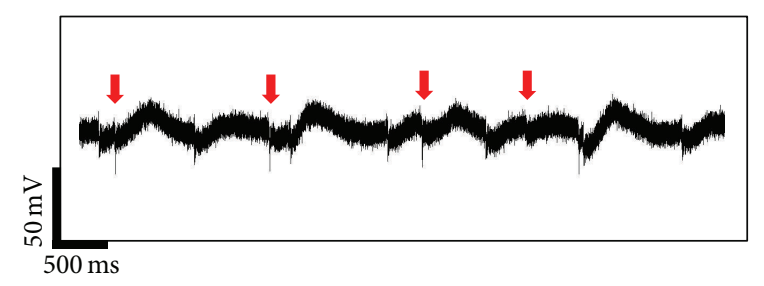

(c)
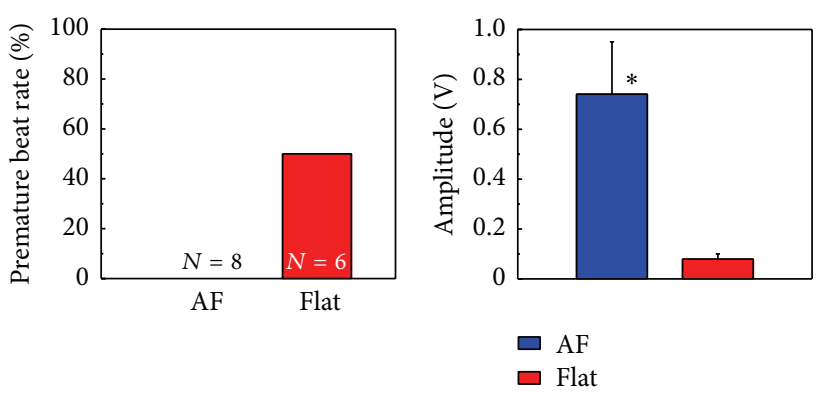

(d)

(e)

FIgURE 4: Extracellular recording of IMR hiPS-cardiomyocytes (CMs) by MEA at day 6. Spontaneous beatings of CMs on aligned fiber- (AF-) covered (a) and gelatin-coated (Flat) MEA (b) at day 6. (c) Premature beating recorded on Flat samples at day 6 . The red arrows mark the representative beating of the premature beating. (d) Premature beating rate of samples at day $6 . N$ is the number of the respective samples. (e) Amplitude of field potential recorded on CMs at day 6 (means \pm s.e., $n=3,{ }^{*} P<0.05$ ).

and Supplementary Figure 4(a)), and after applying Fridericia correction, the cQT intervals showed slight difference between two types of cells (Figure 5(f) and Supplementary Figure 4(d)). The iCell beats faster in Flat samples than those in AF samples on day 6 and day 10 while there are no such differences in IMR hiPSC-CMs (Supplementary Figures 4(e) and 5(g)). Moreover, our data may indicate the best timing for carrying out CMs based drug test which may be at around 6 days, similar to the recommended timing [22]. These data indicated intrinsic differences between IMR hiPSC-CMs and the commercial iCell due to different differentiation methods. The conduction velocity of the spontaneous contraction 


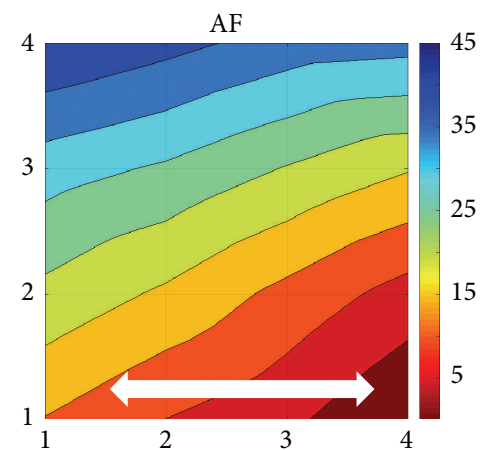

(a)

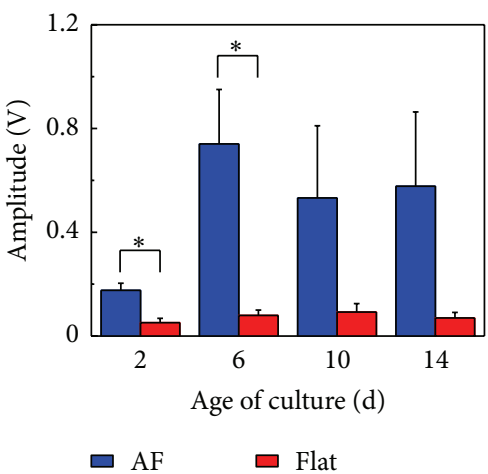

(d)

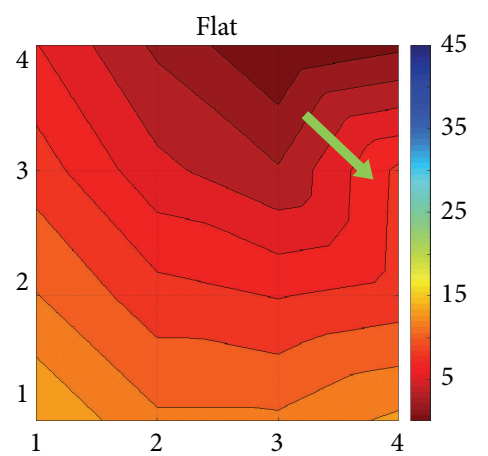

(b)

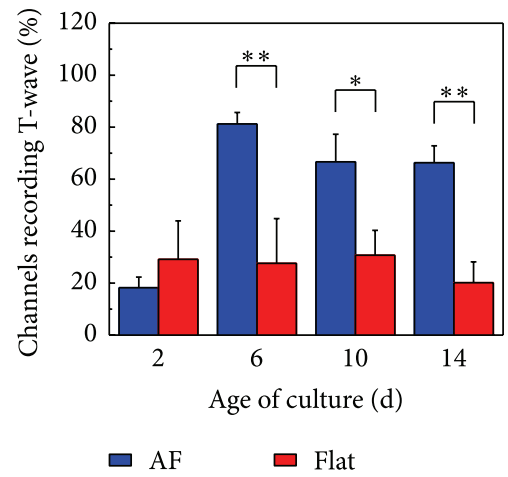

(e)

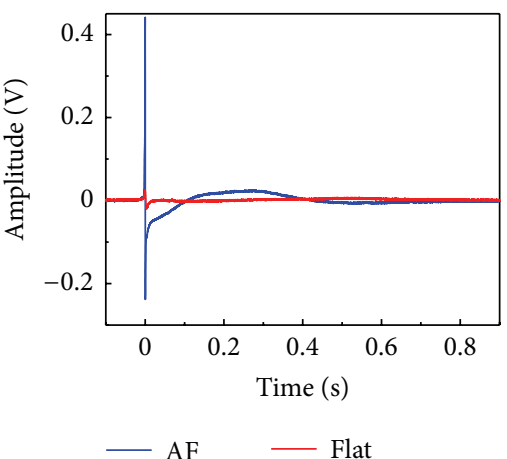

(c)

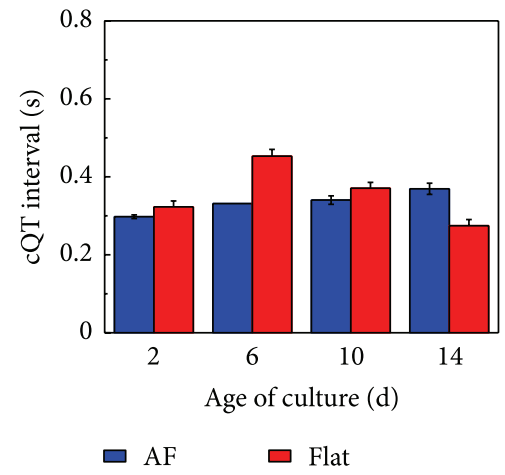

(f)

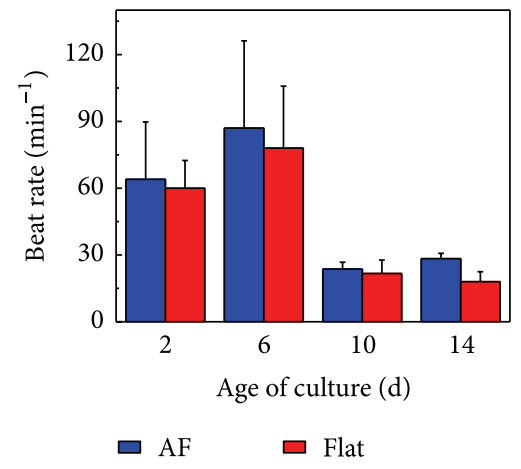

(g)

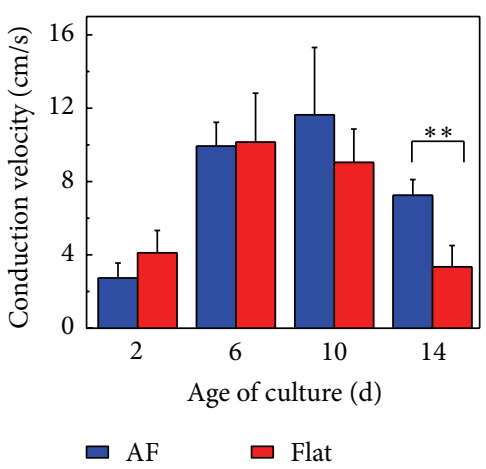

(h)

FIGURE 5: Electrical characterization of IMR hiPS-cardiomyocytes (CMs). (a-b) Activation maps showing the propagation of contraction on day 6. The white arrow marks the fiber orientation and the green arrow marks the delay area. (c) Field potentials (FPs) of CMs cultured on the two types of substrates at day 6. (d) Amplitude of FP at different culture times (means \pm s.e., $n=3,{ }^{*} P<0.05$ ). (e) The ratio of the channels recording the T-wave (means \pm s.e., $n=3,{ }^{*} P<0.05,{ }^{* *} \mathrm{P}<0.01$ ). (f) cQT intervals recorded from CMs at different culture times (means \pm s.e., $n=3$ ). (g) CM beating rate at different culture times (means \pm s.e., $n=3$ ). (h) Conduction velocity of spontaneous contraction propagation at different culture times (means \pm s.e., $n=3,{ }^{* *} P<0.01$ ).

showed no dramatic difference between AF and Flat samples (Figure 5(h), Supplementary Figure 4(f)).

The electrical stimulation has been used to pace hiPSCCMs [23]; here we applied stimulation $( \pm 700 \mathrm{mV}, 5 \mathrm{~ms}$ duration) (Figure 6(a)) to quantitatively compare the propagation speed in different directions; for this experiment, the stimulation and recording electrodes were arranged as shown in Figure 6(a). The time course of the field potential after stimulation showed a uniformly propagation and the field potential propagated from one electrode to another along a pathway originating from the location close to the stimulation electrodes (Figures 6(b) and 6(c)). The propagation speed along the fiber direction was more than twofold larger than that perpendicular to the fiber direction (Figure 6(d)), similar to the observation with other types of anisotropic substrates $[24,25]$. Furthermore, the activation map obtained by $4 \times 4$ electrode array arrangement (Figure 6(e)) showed elliptical isochrones with the AF covered samples but an isotropic 


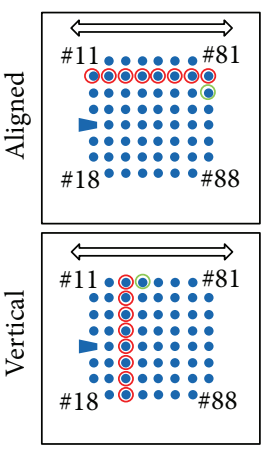

- Stimulation point

$\bigcirc$ Electrode point

(a)

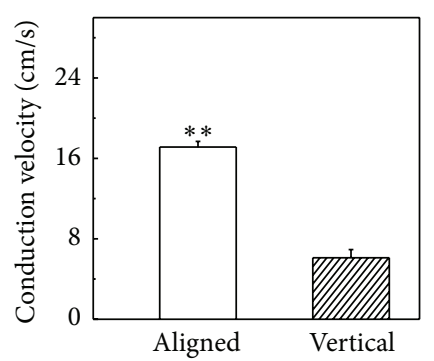

(d)
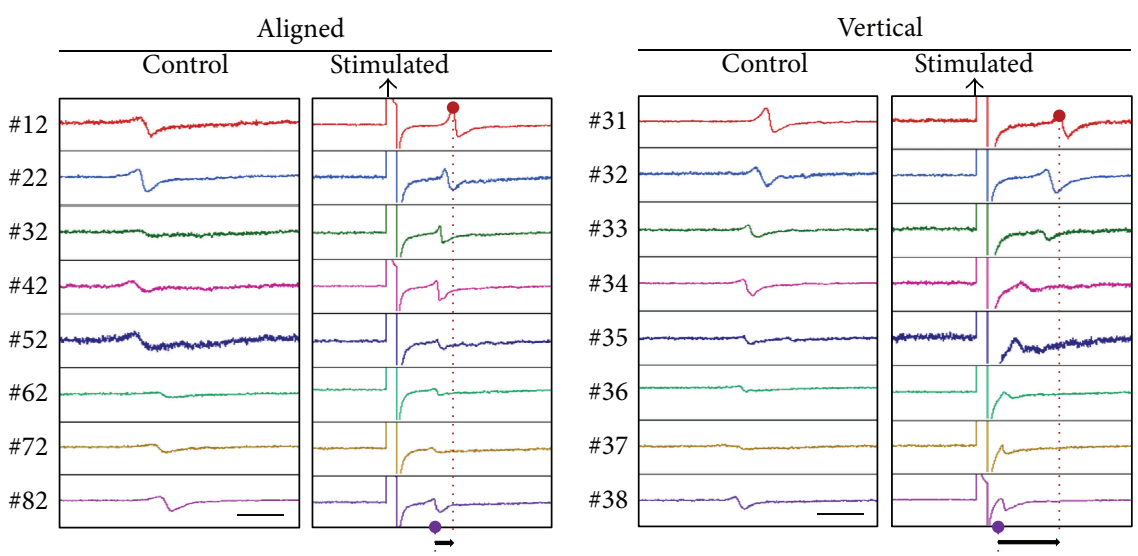

(b)

(c)

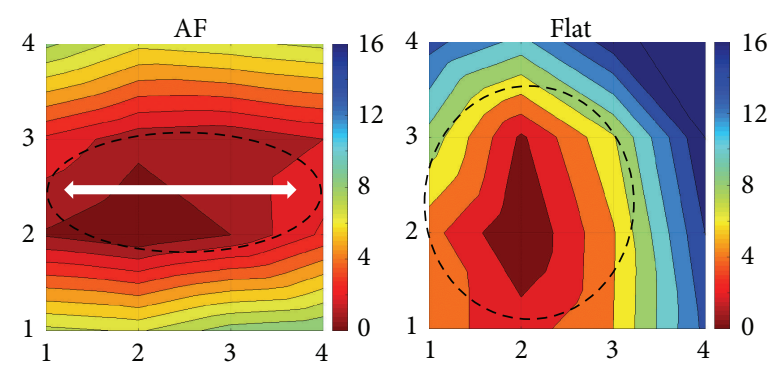

(f)

FIGURE 6: Propagation of the field potential of IMR hiPS-cardiomyocytes. (a) Electrode arrangement for recording and stimulation. The AF orientation is marked by arrow. (b, c) Time course of the field potential along (b) and perpendicular (c) to the fiber direction. The scale bar $=0.2 \mathrm{~s}$. The black arrows mark the starting point of stimulation. (d) Conduction velocity of the stimulated contraction in different directions (means \pm s.e., $n=3,{ }^{* *} P<0.01$ ). (e) Electrode arrangement for activation map analysis. (f) Activation maps showing the propagation of stimulated contraction at day 14 . The color bar from red to blue is linearly divided from $0 \mathrm{~ms}$ to $16 \mathrm{~ms}$. The arrow marks the AF orientation. The encircled line marks the shape of the isochrones of the propagation.

propagation in the Flat samples (Figure 6(f)). In summary, the AF-coated MEA promoted anisotropic cellular organization and anisotropic propagation of the field potential, enhancing the extracellular recording of the cultured CMs. Such an integrated platform thus holds high potential for future studies.

\section{Conclusion}

Electrospun AFs were placed on the surface of a commercial MEA. hiPSC-CMs could be cultured thereon, which resulted in tissue-like CM constructs. The CMs in such a tissue-like constructs were elongated, compact, and matured, showing reduced premature beating, higher signal amplitude, and higher probability of T-wave recording compared with those cultured on the flat substrate without fibers. We therefore confirmed the robustness of AF-coated MEA for the creation of tissue-like constructs of hiPSC-CMs and their reliability for extracellular recording.

\section{Competing Interests}

Kyoto University has applied for a Japanese provisional patent based on the presented research (Li Liu, Junjun Li, Itsunari Minami, Norio Nakatsuji, and Yong Chen).

\section{Authors' Contributions}

Li Liu, Junjun Li, Norio Nakatsuji, and Yong Chen conceived the project; Li Liu and Junjun Li designed the experiments; Junjun Li, Itsunari Minami, Leqian Yu, Kiyotaka Tsuji, Minako Nakajima, Jing Qiao, Masato Suzuki, Ken Shimono, Hitetoshi Kotera, and Li Liu carried out the experiments; Junjun Li, Itsunari Minami, Leqian Yu, Kiyotaka Tsuji, Hitetoshi Kotera, and Li Liu analyzed the data; and Junjun Li, Li Liu, and Yong Chen wrote the paper.

\section{Acknowledgments}

Funding was provided by the Japan Society for the Promotion of Science (JSPS): Grants-in-Aid for Scientific Research 
(B) $(15 \mathrm{H} 03948,26289065)$, Grants-in-Aid for Scientific Research (C) (15K08270), Challenging Exploratory Research (26630096), and Grants-in-Aid for JSPS Fellows (26.04046). Funding was also provided by the Center of Innovation (COI) Program from MEXT and JST, the European Commission through contract no. 604263 (Neuroscaffolds) and the French National Research Agency (ANR) through contract no. ANR12-RPIB-0015 (Cardiac Patch). The WPI-iCeMS is supported by the World Premier International Research Centre Initiative (WPI), MEXT, Japan.

\section{References}

[1] I. Minami, K. Yamada, T. G. Otsuji et al., "A small molecule that promotes cardiac differentiation of human pluripotent stem cells under defined, cytokine- and xeno-free conditions," Cell Reports, vol. 2, no. 5, pp. 1448-1460, 2012.

[2] P. Liang, F. Lan, A. S. Lee et al., "Drug screening using a library of human induced pluripotent stem cell-derived cardiomyocytes reveals disease-specific patterns of cardiotoxicity," Circulation, vol. 127, no. 16, pp. 1677-1691, 2013.

[3] E. G. Navarrete, P. Liang, F. Lan et al., "Screening drug-induced arrhythmia using human induced pluripotent stem cell-derived cardiomyocytes and low-impedance microelectrode arrays," Circulation, vol. 128, no. 11, supplement 1, pp. S3-S13, 2013.

[4] S. R. Braam, L. Tertoolen, A. van de Stolpe, T. Meyer, R. Passier, and C. L. Mummery, "Prediction of drug-induced cardiotoxicity using human embryonic stem cell-derived cardiomyocytes," Stem Cell Research, vol. 4, no. 2, pp. 107-116, 2010.

[5] E. Matsa, D. Rajamohan, E. Dick et al., "Drug evaluation in cardiomyocytes derived from human induced pluripotent stem cells carrying a long QT syndrome type 2 mutation," European Heart Journal, vol. 32, no. 8, pp. 952-962, 2011.

[6] A. Mathur, Z. Ma, P. Loskill, S. Jeeawoody, and K. E. Healy, "In vitro cardiac tissue models: current status and future prospects," Advanced Drug Delivery Reviews, vol. 96, pp. 203-213, 2016.

[7] D.-H. Kim, Kshitiz, R. R. Smith et al., "Nanopatterned cardiac cell patches promote stem cell niche formation and myocardial regeneration," Integrative Biology, vol. 4, no. 9, pp. 1019-1033, 2012.

[8] L. Wang, L. Liu, X. Li, N. Magome, K. Agladze, and Y. Chen, "Multi-electrode monitoring of guided excitation in patterned cardiomyocytes," Microelectronic Engineering, vol. 111, pp. 267271, 2013.

[9] A. Chen, D. K. Lieu, L. Freschauf et al., "Shrink-film configurable multiscale wrinkles for functional alignment of human embryonic stem cells and their cardiac derivatives," Advanced Materials, vol. 23, no. 48, pp. 5785-5791, 2011.

[10] Y.-D. Lin, M.-C. Ko, S.-T. Wu et al., "A nanopatterned cellseeded cardiac patch prevents electro-uncoupling and improves the therapeutic efficacy of cardiac repair," Biomaterials Science, vol. 2, no. 4, pp. 567-580, 2014.

[11] Y. Li, G. Huang, X. Zhang et al., "Engineering cell alignment in vitro," Biotechnology Advances, vol. 32, no. 2, pp. 347-365, 2014.

[12] I. C. Parrag, P. W. Zandstra, and K. A. Woodhouse, "Fiber alignment and coculture with fibroblasts improves the differentiated phenotype of murine embryonic stem cell-derived cardiomyocytes for cardiac tissue engineering," Biotechnology and Bioengineering, vol. 109, no. 3, pp. 813-822, 2012.
[13] S. S. Nunes, J. W. Miklas, J. Liu et al., "Biowire: a platform for maturation of human pluripotent stem cell-derived cardiomyocytes," Nature Methods, vol. 10, no. 8, pp. 781-787, 2013.

[14] Y. Orlova, N. Magome, L. Liu, Y. Chen, and K. Agladze, "Electrospun nanofibers as a tool for architecture control in engineered cardiac tissue," Biomaterials, vol. 32, no. 24, pp. 5615-5624, 2011.

[15] I. Itzhaki, L. Maizels, I. Huber et al., "Modelling the long QT syndrome with induced pluripotent stem cells," Nature, vol. 471, no. 7337, pp. 225-230, 2011.

[16] Z. Ma, M. Kotaki, R. Inai, and S. Ramakrishna, "Potential of nanofiber matrix as tissue-engineering scaffolds," Tissue Engineering, vol. 11, no. 1-2, pp. 101-109, 2005.

[17] I. Shabani, V. Haddadi-Asl, E. Seyedjafari, F. Babaeijandaghi, and M. Soleimani, "Improved infiltration of stem cells on electrospun nanofibers," Biochemical and Biophysical Research Communications, vol. 382, no. 1, pp. 129-133, 2009.

[18] K. Nakao, W. Minobe, R. Roden, M. R. Bristow, and L. A. Leinwand, "Myosin heavy chain gene expression in human heart failure," Journal of Clinical Investigation, vol. 100, no. 9, pp. 2362-2370, 1997.

[19] G. Meiry, Y. Reisner, Y. Feld et al., "Evolution of action potential propagation and repolarization in cultured neonatal rat ventricular myocytes," Journal of Cardiovascular Electrophysiology, vol. 12, no. 11, pp. 1269-1277, 2001.

[20] I. Kehat, L. Khimovich, O. Caspi et al., "Electromechanical integration of cardiomyocytes derived from human embryonic stem cells," Nature Biotechnology, vol. 22, no. 10, pp. 1282-1289, 2004.

[21] S. Kadota, I. Minami, N. Morone, J. E. Heuser, K. Agladze, and N. Nakatsuji, "Development of a reentrant arrhythmia model in human pluripotent stem cell-derived cardiac cell sheets," European Heart Journal, vol. 34, no. 15, pp. 1147-1156, 2013.

[22] Y. Sekino, K. Sato, Y. Kanda, and S. Ishida, "Developing and standardizing experimental protocols using human iPS-derived cells to predict adverse drug reactions in pre-clinical safety studies," Bulletin of National Institute of Health Sciences, no. 131, pp. 25-34, 2013.

[23] D. Hernández, R. Millard, P. Sivakumaran et al., "Electrical stimulation promotes cardiac differentiation of human induced pluripotent stem cells," Stem Cells International, vol. 2016, Article ID 1718041, 12 pages, 2016.

[24] V. G. Fast and A. G. Kléber, "Anisotropic conduction in monolayers of neonatal rat heart cells cultured on collagen substrate," Circulation Research, vol. 75, no. 3, pp. 591-595, 1994.

[25] N. Bursac, K. K. Parker, S. Iravanian, and L. Tung, "Cardiomyocyte cultures with controlled macroscopic anisotropy: a model for functional electrophysiological studies of cardiac muscle," Circulation Research, vol. 91, no. 12, pp. e45-e54, 2002. 

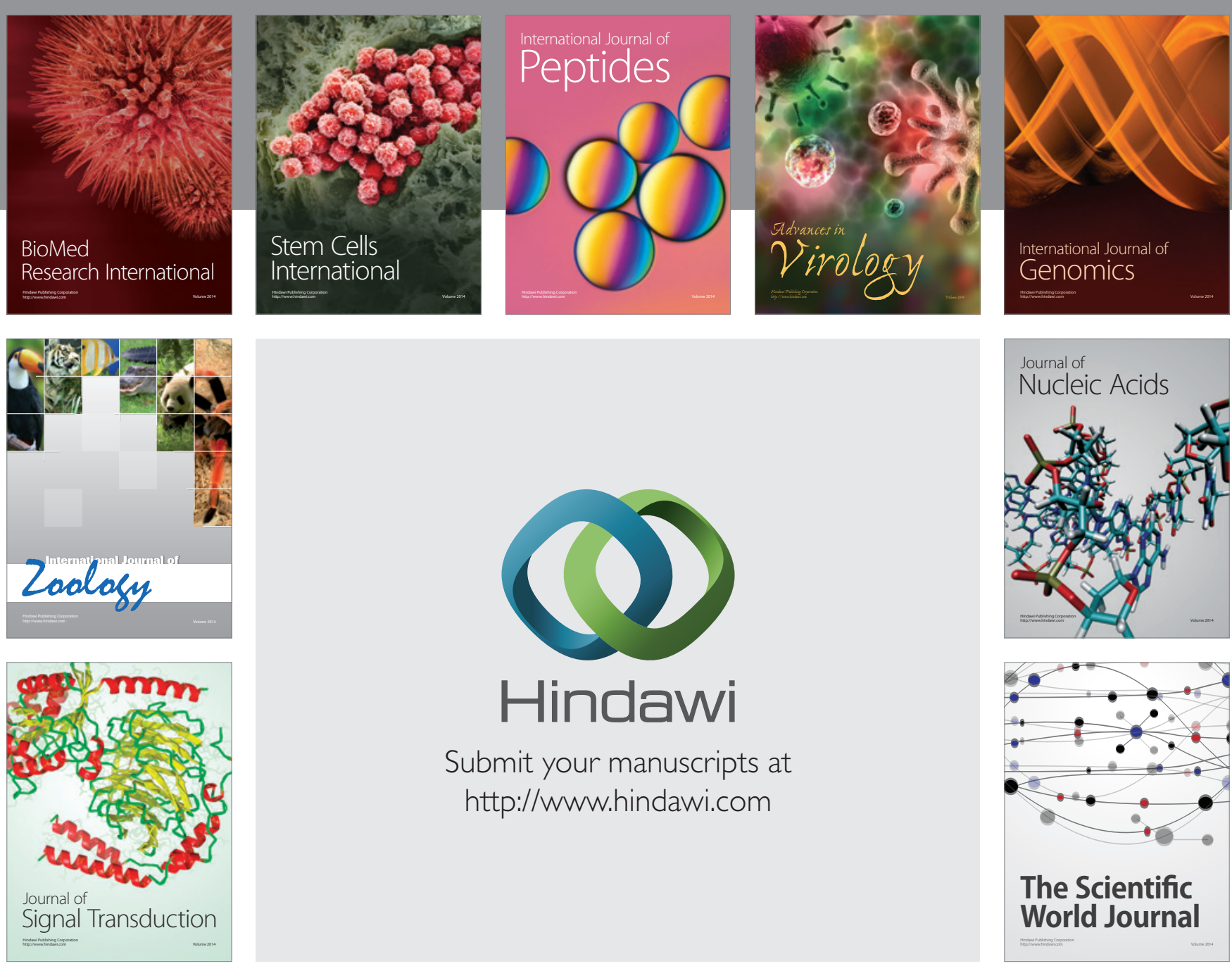

Submit your manuscripts at

http://www.hindawi.com
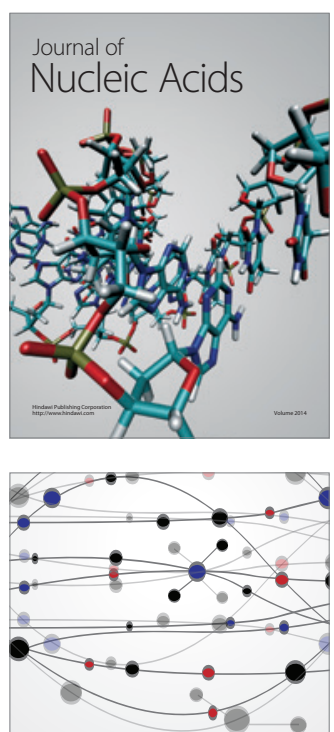

The Scientific World Journal
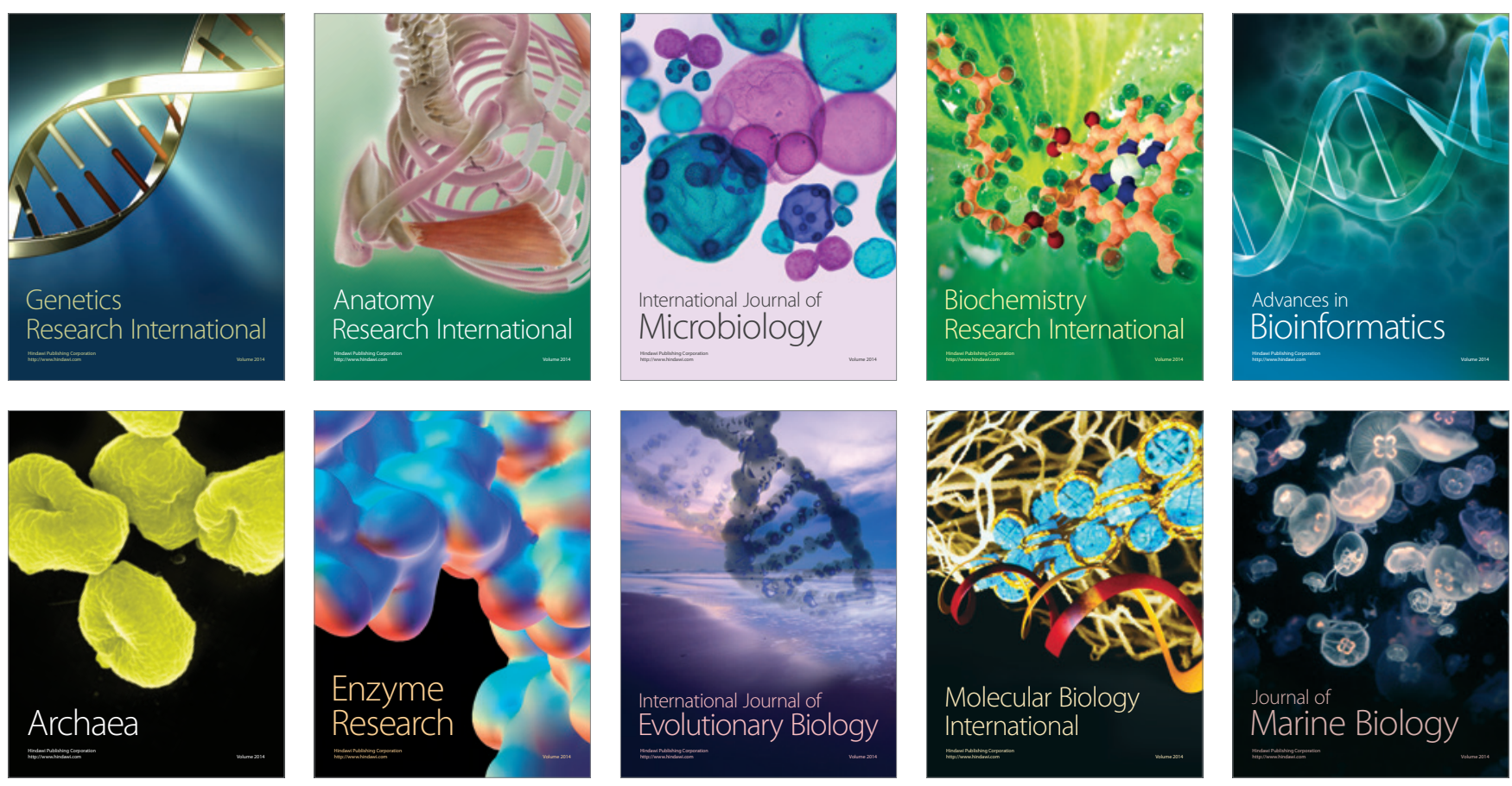